\title{
Parálisis facial, causas y tratamiento quirúrgico en un centro de referencia en cirugía plástica y reconstructiva en México
}

\author{
Facial paralysis, etiology and surgical treatment in a tertiary care center in plastic and \\ reconstructive surgery in Mexico
}

\section{Enrique Chávez-Serna', José E. Telich-Tarriba², Carlos Altamirano-Arcos², Luciano Nahas-Combina² y Alexander Cárdenas-Mejía ${ }^{2,3 *}$}

${ }^{1}$ Servicio de Cirugía General, División de Cirugía General, Hospital de Especialidades, Centro Médico Nacional Siglo XXI, División de Estudios de Posgrado, Universidad Nacional Autónoma de México; ${ }^{2}$ Servicio de Cirugía Plástica y Reconstructiva, División de Cirugía Plástica y Reconstructiva, Hospital General Dr. Manuel Gea González, División de Estudios de Posgrado, Universidad Nacional Autónoma de México; ${ }^{3}$ Servicio de Clínica de Nervio Periférico, División de Cirugía Plástica y Reconstructiva, Hospital General Dr. Manuel Gea González. Ciudad de México, México

\section{Resumen}

Antecedentes: La parálisis facial es una afección incapacitante que con frecuencia causa un impacto negativo en los aspectos cosméticos, funcionales, sociales, psicológicos y económicos. El tratamiento quirúrgico tiene como objetivo mejorar la calidad de vida dejando la menor cantidad posible de secuelas. Objetivo: Describir la experiencia del manejo quirúrgico y proponer un algoritmo de tratamiento. Método: Se realizó un estudio retrospectivo, de 2017 a 2019, de los expedientes de pacientes con afección del nervio facial. Las variables consideradas fueron la edad, el sexo, la etiología, el lado afectado y los procedimientos quirúrgicos realizados. Resultados: Se obtuvieron 108 pacientes y la causa más frecuente fue la parálisis facial del desarrollo sindrómica (42\%), seguida de la resección de tumores intracraneales (29\%). Se realizaron 225 procedimientos, con un promedio de 2.7 por paciente. El procedimiento dinámico más realizado fue el colgajo libre de gracilis (59\%). Los procedimientos estáticos más frecuentes fueron la colocación de una pesa de oro (24\%) y la recreación del surco nasogeniano (13\%). Conclusiones: La secuencia del tratamiento quirúrgico dependerá del tiempo de evolución, la etiología, los nervios disponibles y el estado de la musculatura facial. El colgajo libre de gracilis continúa siendo el procedimiento de elección en la reconstrucción de la parálisis facial. Adicionalmente se requieren procedimientos estáticos para lograr un resultado estético y funcional satisfactorio.

Palabras clave: Parálisis facial. Parálisis facial del desarrollo. Tercer nivel de atención. Cirugía reconstructiva. Tratamiento quirúrgico. Síndrome de Moebius.

\begin{abstract}
Background: Facial paralysis is a frequent disabling entity that causes a negative impact on the cosmetic, functional, social, psychological and economic aspects of the patient. Surgical treatment aims to restore the patient to her previous life with the
\end{abstract}

\footnotetext{
Correspondencia:

*Alexander Cárdenas-Mejía

Calzada de Tlalpan 4800

Belisario Domínguez Sección 16

Fecha de recepción: 19-08-2020

C.P. 14080, Ciudad de México, México Fecha de aceptación: 20-10-2020

E-mail: alexcardenas@ hotmail.com

DOI: 10.24875/CIRU.20000916

Cir Cir. 2021;89(6):718-727

Contents available at PubMed

www.cirugiaycirujanos.com

0009-7411/@ 2020 Academia Mexicana de Cirugía. Publicado por Permanyer. Este es un artículo open access bajo la licencia CC BY-NC-ND (http://creativecommons.org/licenses/by-nc-nd/4.0/).
} 
fewest possible sequelae. Objective: Describe the experience of surgical management and propose a treatment algorithm. Method: A retrospective study was carried out from 2017 to 2019 of the records of patients with facial nerve involvement. The variables were age, sex, etiology, affected side and procedures performed. Results: 108 patients were obtained. The most frequent cause was development facial paralysis $(41,8 \%)$, followed by resection of intracranial tumors (29\%). A total of 225 procedures were performed, average per patient of 2.7. The most performed dynamic procedure was the gracilis-free flap (59\%). The most frequent static procedures were the placement of a gold weight (24\%) and the recreation of the nasogenian sulcus (13\%). Conclusions: The surgical treatment algorithm will depend on the evolution time, etiology, donor nerves and state of the facial musculature. The gracilis-free flap continues to be the gold standard procedure in facial paralysis reconstruction. Static procedures are additionally required to achieve a satisfactory aesthetic and functional result.

Key words: Facial paralysis. Developmental facial paralysis. Tertiary care center. Reconstructive surgery. Surgical treatment. Moebius syndrome.

\section{Introducción}

La parálisis facial periférica es un trastorno nervioso que presenta varias etiologías y se caracteriza por la afectación de las vías motoras y sensitivas del séptimo nervio craneal. La parálisis facial idiopática, o parálisis de Bell, continúa siendo la causa más frecuente de parálisis facial en el mundo, con una incidencia de 13 a 34 casos por cada 10,000 personas al año en los Estados Unidos de América ${ }^{1}$ y de 20 a 30 casos por 100,000 personas al año en México². Otras causas menos frecuentes son parálisis facial traumática, neoplásica, iatrogénica, del desarrollo y congénita.

El enfoque terapeútico de un paciente con parálisis facial debe ser integral y multidisciplinario. Clásicamente, el diagnóstico de la parálisis facial, independientemente de la etiología, ha sido clínico incluyendo una minuciosa anamnesis y una completa exploración física. En la anamnesis se deben enfatizar varios aspectos: posible causa, tiempo de evolución, síntomas y preocupaciones del paciente. La exploración física se debe enfocar en la competencia oral, el estado de la córnea y el patrón de movilidad facial. Cada ramo del nervio facial debe evaluarse individualmente:

- Frontotemporal: elevación de cejas.

- Cigomático: cierre palpebral.

- Bucal: sonrisa y silbido.

- Mandibular-marginal: depresión del labio inferior.

Debe complementarse con la evaluación de otros pares craneales y un examen de la motilidad ocular extrínseca (III, IV y VI pares). Existen diversas escalas que clasifican la gravedad de la parálisis facial, pero la más aceptada globalmente por considerar solo aspectos clínicos y ser accesible y fácil de reproducir es la escala de House-Brackmann, que evalúa el grado de disfunción al realizar movimientos voluntarios; sin embargo, existen otras escalas que evalúan el estado del pacientes antes y después de la cirugía con registros videográficos, que son más objetivas $^{3,4}$.

Los estudios neurofisiológicos son una herramienta indispensable para realizar un diagnóstico certero y poder determinar el procedimiento quirúrgico ideal para cada paciente. Evalúan el grado de afectación del nervio facial y la integridad de la musculatura facial. Entre los más accesibles se encuentra la electromiografía, que determina el número de fibras musculares reclutables por territorio registrando potenciales de acción y actividad eléctrica de los músculos faciales ${ }^{5,6}$. Es útil en pacientes con parálisis facial de mayor tiempo evolutivo, cuando ha finalizado el proceso denervativo, y permite identificar potenciales de unidad motora polifásica (potenciales de reinervación). En todos los pacientes se deberá realizar una electromiografía previa a los procedimientos quirúrgicos ${ }^{4,7}$.

El tratamiento quirúrgico y multidisciplinario de la parálisis facial se basa en la importancia de la expresión facial para el desarrollo del individuo en todas sus esferas psicosociales. La parálisis facial es percibida negativamente en el aspecto social y afecta funciones esenciales como la protección corneal, la respiración nasal, la competencia labial y el habla. La sonrisa constituye un gesto trascendental y cuando se ve afectada se asocia directamente con depresión, aislamiento y disminución significativa de la calidad de vida ${ }^{8,9}$.

En general, las técnicas de reconstrucción en parálisis facial se clasifican en procedimientos estáticos y dinámicos. Históricamente, los procedimientos estáticos fueron considerados la primera opción en el tratamiento quirúrgico de la parálisis facial; tienen como objetivo reposicionar los tejidos blandos, contrarrestando los efectos de la gravedad y teniendo 
como resultado final una simetría facial en reposo ${ }^{10}$. Sin embargo, los procedimientos estáticos no aportan movilidad espontánea ni movimientos voluntarios simétricos. Por su parte, los procedimientos dinámicos aportan movilidad, favorecen los movimientos voluntarios espontáneos y las expresiones faciales simétricas, y en la actualidad son el método de referencia para restablecer los movimientos en el rostro paralizado ${ }^{10,11}$.

Los procedimientos dinámicos tienden a ser más exitosos y fructíferos que los estáticos, y deben ofrecerse a todo paciente que considere la reconstrucción, a menos que existan contraindicaciones de riesgo para la salud que no permitan realizarlos. Los enfoques más comunes para la reconstrucción dinámica, además de la neurorrafia, los injertos nerviosos y las transferencias nerviosas, incluyen la transferencia muscular regional y la transferencia muscular libre.

En México, la experiencia reportada en cirugía reconstructiva en parálisis facial es escasa, ya que pocos centros en el país realizan este tipo de procedimientos, además de que no se cuenta con un algoritmo de manejo establecido. El número de procedimientos, en promedio, que requiere un paciente no está descrito, ni los más frecuentemente realizados para la reanimación facial. El objetivo de este artículo es describir la experiencia de la clínica de nervio periférico y parálisis facial del Hospital General Dr. Manuel Gea González, centro de referencia en cirugía plástica y reconstructiva en la Ciudad de México, así como proponer un algoritmo de tratamiento quirúrgico.

\section{Método}

Diseñamos un estudio retrospectivo transversal y descriptivo en el cual se incluyeron todos los pacientes sometidos a cirugía de reanimación por parálisis facial en el periodo de enero de 2017 a diciembre de 2019. La información se extrajo de los expedientes clínicos y fotográficos de los pacientes, y fue ingresada en una hoja de captura de datos en la que se incluyeron variables demográficas de los pacientes, etiología de la parálisis, lado afectado, cantidad y tipo de procedimientos quirúrgicos. Se realizó un análisis descriptivo de las características de la población con el paquete estadístico SPSS versión 25, en el cual las variables categóricas se reportan en porcentajes y las continuas como promedio ( \pm desviación estándar [DE]) o mediana (rango intercuartílico) de acuerdo con su distribución.
Tabla 1. Características demográficas de los pacientes y hemicara afectada

\begin{tabular}{lcc}
\hline Variable & $\mathbf{n}(\%)$ & Promedio \\
\hline Sexo: & $45(42)$ & - \\
Masculino & $63(58)$ & - \\
Femenino & Rango: 1 a 89 & $29(\mathrm{DE}: 21)$ \\
Edad & & \\
Hemicara afectada & $49(45)$ & - \\
$\quad$ Derecha & $36(33)$ & - \\
Izquierda & $23(21)$ & - \\
\hline Bilateral & &
\end{tabular}

DE: desviación estándar.

\section{Resultados}

Se incluyeron 108 pacientes, 63 (58\%) mujeres y $45(42 \%)$ hombres. La edad promedio al momento de la cirugía fue de 28,8 años (DE: 21), con un rango de 1 a 89 años (Tabla 1). El lado con más frecuencia afectado fue el derecho, con 49 casos (45\%); el lado izquierdo representó 36 casos (33\%) y la afectación fue bilateral en 23 casos (21\%).

En cuanto a la etiología, la causa más observada en nuestro servicio fue la parálisis facial del desarrollo sindrómica, con 46 casos (42\%); las causas iatrogénicas representaron el segundo lugar, debido a resección de tumores intracraneales, con 32 casos $(29 \%)$, y en tercer lugar, la parálisis facial idiopática o de Bell, en 18 casos (16\%).

Otras causas menos frecuentes fueron traumáticas en seis casos (6\%), neoplasias que por su localización y crecimiento comprometieron el nervio facial en tres casos (3\%), e infecciosas (Ramsay-Hunt, tuberculosis meníngea) en cuatro casos (4\%) (Fig. 1).

Dentro de la parálisis del desarrollo sindrómica, el síndrome de Moebius representó la mayoría de los casos, en 43 pacientes (88\%), seguido de la microsomía hemifacial en tres casos (6\%) y de otras causas menos frecuentes, como síndrome de Goldenhar en dos casos (4\%) y secuencia CHARGE (coloboma ocular, cardiopatía, atresia de coanas, retraso del crecimiento o del desarrollo, hipoplasia genital y anomalías del pabellón auricular o sordera) en un caso $(2 \%)$. Dentro del espectro del síndrome de Moebius, la variante clásica o completa representó la mayoría, con 24 casos $(56 \%)$, seguida de la variante incompleta, con 13 casos (30\%), y por último de la variante Moebius-like, con seis casos (14\%) (afección de otros pares craneales) (Figs. 2 y 3 ). 


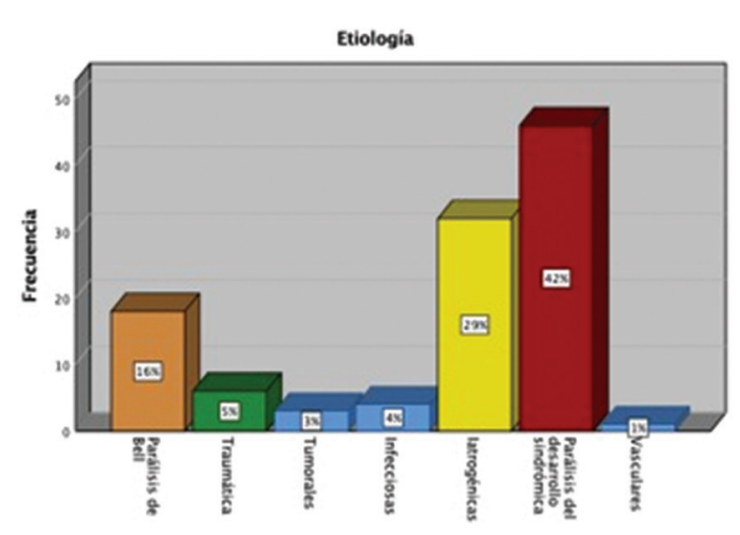

Figura 1. Etiología de la parálisis facial.

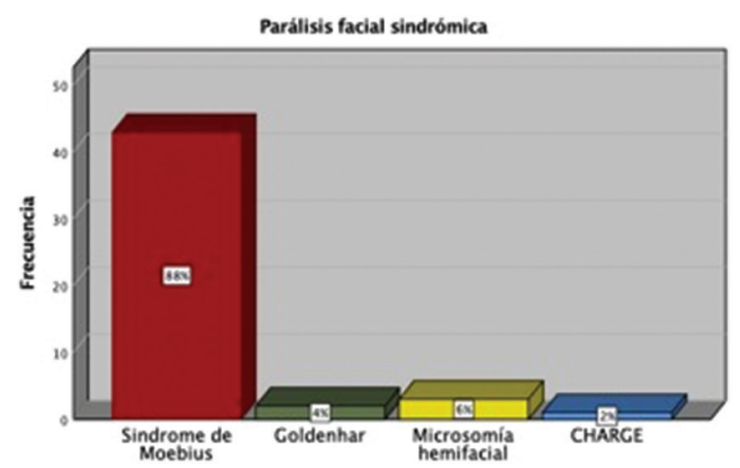

Figura 2. Parálisis facial del desarrollo sindrómica.

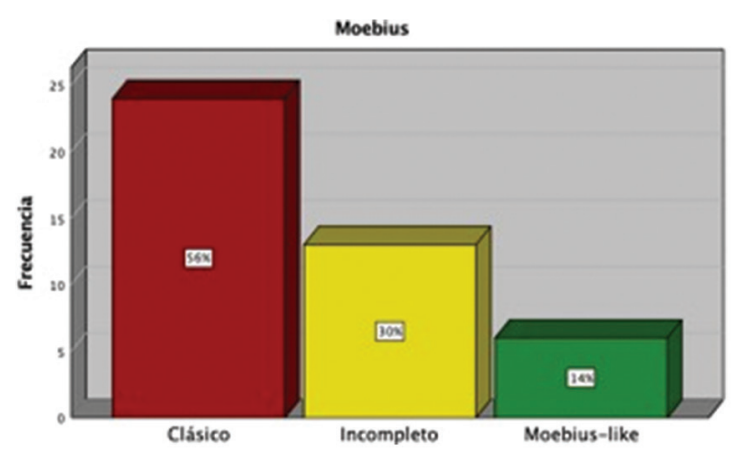

Figura 3. Síndrome de Moebius.

Dentro de las causas iatrogénicas se integran las resecciones de tumores intracraneales e intratemporales, de los cuales los más frecuentes fueron schwannomas (38\%), colesteatomas resecados por mastoidectomía radical (22\%), neurinomas acústicos $(13 \%)$, meningiomas $(13 \%)$, linfangiomas $(3 \%)$, paragangliomas $(3 \%)$, gliomas en el tallo encefálico $(3 \%)$, neurofibromas $(3 \%)$ y craneofaringiomas $(3 \%)$.
Tabla 2. Procedimientos quirúrgicos realizados

\begin{tabular}{lcc}
\hline Variable & Promedio & DE \\
\hline Número de procedimientos & 2.7 & 2.1 \\
Procedimientos dinámicos & 1.8 & 0.6 \\
Procedimientos estáticos & 2.1 & 2 \\
\hline DE: desviación estándar. & &
\end{tabular}

DE: desviación estándar.

Se realizó en total 225 procedimientos, de los cuales $122(54 \%)$ fueron dinámicos y 103 (46\%) fueron estáticos. El promedio de procedimientos que se realizó en cada paciente fue de 2.7 (DE: 2,1; rango: 1-16) (Tabla 2).

El procedimiento dinámico más realizado fue el colgajo libre de músculo gracilis, con 51 (59\%) colgajos realizados, de los cuales 31 (34\%) fueron unilaterales y $23(25 \%)$ bilaterales. Los unilaterales se realizaron en dos tiempos: el primer tiempo consistió en realizar un injerto nervioso cruzado del nervio bucal sano al surco nasogeniano de la hemicara afectada usando el nervio sural como injerto, y en el segundo tiempo, 4 a 6 meses después, se realizó un colgajo libre de gracilis doble inervado con coaptación término-terminal del obturador del gracilis al injerto nervioso cruzado y de manera término-lateral al nervio maseterino homolateral. La mayoría de los colgajos bilaterales se realizaron en dos tiempos, con un tiempo promedio entre ellos de 1 a 6 meses; en seis casos se hizo en un solo tiempo.

El injerto nervioso cruzado representó el segundo procedimiento dinámico que se realizó con mayor frecuencia, en 15 casos (16\%). Tiene como objetivo reanimar la hemicara afectada en lesiones con menos de 6 meses de evolución El más frecuente de esta serie fue la coaptación entre los nervios bucal-bucal en nueve casos $(7 \%)$, seguido de la coaptación marginal-marginal en cinco casos (4\%) y marginal-hipogloso en un caso (1\%).

Cuando fallan los procedimientos dinámicos libres, los procedimientos dinámicos regionales son una alternativa válida que se realiza con frecuencia. En nuestro servicio, el procedimiento de Viterbo se realizó en 11 casos (12\%) con falla en el colgajo de gracilis. Otros procedimientos realizados con menor frecuencia fueron las transferencias nerviosas, en siete casos $(8 \%)$, de las cuales el procedimiento de "niñera» (baby-sitter) fue el más frecuente, en cuatro casos, seguido de las transferencias maseterino-bucal en dos casos y de las transferencias hipogloso-marginal y 
espinal-marginal en un caso cada una. La neurorrafia primaria se llevó a cabo en cuatro casos (4\%), cuando el paciente se presentó dentro de las primeras 72 horas posteriores a la lesión, generalmente debido a causas traumáticas (Fig. 4).

Los procedimientos estáticos que se realizaron con mayor frecuencia fueron, en primer lugar, la colocación de una pesa de oro en 25 casos (24\%); en segundo lugar, la recreación del surco nasogeniano o comisuroplastia en 13 casos (13\%); en tercer lugar, la tira tarsal en 11 casos (11\%); y por último, la neurotización de músculos depresores en 10 casos (10\%). Otros procedimientos menos frecuentes fueron la suspensión de la ceja, la lipoinyección facial, la suspensión del párpado inferior, la ritidectomía asimétrica, la plicatura del músculo orbicular, la miotomía del músculo frontal y la blefaroplastia asimétrica; y dentro de los no quirúrgicos, la aplicación de toxina botulínica y la rehabilitación con terapia de biorretroalimentación (biofeedback) (Fig. 5).

\section{Discusión}

En la actualidad, gracias a los avances en las técnicas quirúrgicas de parálisis facial, además de restablecer la función, como objetivo principal en el tratamiento quirúrgico se incluye la restauración simétrica, voluntaria y espontánea de la dinámica facial. Cada procedimiento deberá estar dirigido a la zona afectada que se decide reanimar: labio inferior, comisura oral, porción media de la cara, región ocular 0 porción frontal. El tratamiento oportuno y multidisciplinario ha mejorado considerablemente la calidad de vida de los pacientes ${ }^{12}$.

En nuestro estudio se encontró que la parálisis facial del desarrollo sindrómica es la causa más frecuente de parálisis facial, y dentro de la etiología sindrómica, el síndrome de Moebius fue la causa más frecuente, siendo la variante clásica o completa la más común. Esto contrasta con la información publicada en otras series y se debe a que nuestro hospital es centro de referencia del país para malformaciones craneofaciales congénitas ${ }^{13-15}$. Gousheh y Arasteh $^{16}$ realizaron un estudio retrospectivo de 655 pacientes con parálisis facial que arrojó resultados similares a los nuestros, siendo la parálisis facial sindrómica la más frecuente.

Terzis y Konofaos ${ }^{17}$, en una serie de 166 pacientes con parálisis facial secundaria a la resección de tumores intracraneales, reportaron el neurinoma acústico como la principal causa, seguido de los tumores

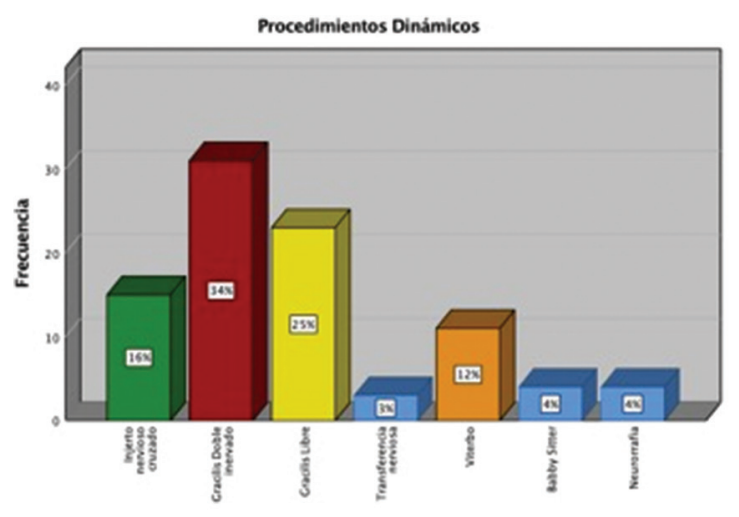

Figura 4. Procedimientos dinámicos.

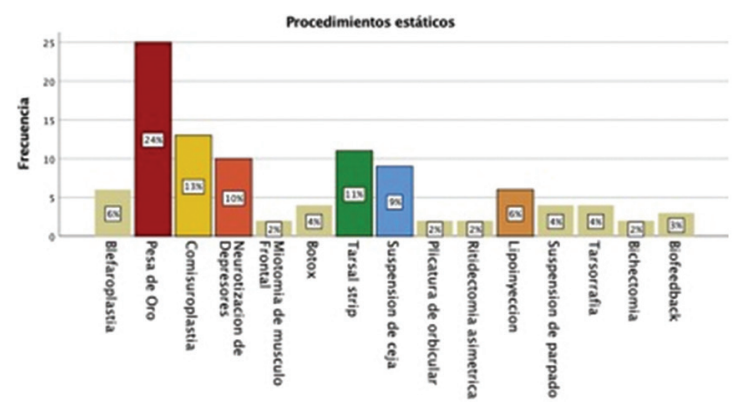

Figura 5. Procedimientos estáticos.

extratemporales, como los hemangiomas, y por último de los tumores intratemporales, como los colesteatomas. En nuestro estudio, la resección de tumores intracraneales y extracraneales fue la segunda causa más frecuente de parálisis facial. Los schwannomas fueron los más comunes, seguidos de la mastoidectomía para abordaje de colesteatomas, los meningiomas y los neurinomas acústicos. La alta incidencia de parálisis facial secundaria a resección de tumores en nuestro centro se debe posiblemente a la referencia interhospitalaria que se realiza entre el Instituto Nacional de Neurología y Neurocirugía.

La parálisis facial idiopática (Bell) continúa siendo la causa más frecuente de parálisis facial en el mundo, y afortunadamente en un $60-80 \%$ de los casos se produce una recuperación completa y espontánea al cabo de un par de meses ${ }^{18}$. Sin embargo, los pacientes que cursan con una evolución tórpida o parálisis facial grave deberán ser referidos de manera temprana a centros con experiencia en cirugía reconstructiva de parálisis facial, ya que en su mayoría requerirán tratamiento quirúrgico escalonado. En la mayoría de los centros en donde se realiza cirugía de parálisis 
facial se reporta a la parálisis de Bell como la causa más frecuente de parálisis facial que requiere tratamiento quirúrgico ${ }^{19,20}$, en contraste con nuestros resultados, en los que la parálisis de Bell representó el tercer lugar, probablemente debido a que la mayoría de los pacientes que cursan con secuelas desconocen la existencia del tratamiento quirúrgico, pues muy pocos centros realizan este tipo de cirugías en el país. Por otro lado, los pacientes que cursan con parálisis facial aguda de Bell son referidos al servicio de otorrinolaringología para manejo médico inicial, y solo los casos con secuelas después de 6 meses son referidos a nuestro servicio para abordaje quirúrgico.

Otras causas no tan frecuentes en nuestra serie fueron las traumáticas, en su mayoría secundarias a heridas por arma blanca o a fracturas del macizo facial, con sección parcial o completa de alguno de los ramos del nervio facial. A pesar de que llega un elevado número de pacientes con heridas traumáticas ${ }^{13}$, las lesiones del nervio facial no son tan frecuentes en esta serie. En un $20 \%$ de los pacientes que presentan parálisis facial tardía por traumatismos cerrados se producirá una recuperación completa, ya que es secundaria a edema y tendrán buen pronóstico; al contrario, si la parálisis se presenta en el momento de la lesión, lo más probable es que no exista recuperación, como sucede en los traumatismos penetrantes, y se requiera tratamiento quirúrgico ${ }^{21-23}$.

En todos los casos de esta serie se realizaron procedimientos quirúrgicos, excepto en dos pacientes con síndrome de Moebius en los que debido a su edad no se realizó ningún procedimiento, y un paciente con parálisis de Bell que no fue candidato a tratamiento quirúrgico se envió a terapia de rehabilitación con biofeedback. El promedio de procedimientos realizados en cada paciente fue de 2.7, incluyendo estáticos y dinámicos. En la mayoría de las series publicadas, los pacientes son sometidos como mínimo a un promedio de dos cirugías para lograr una reanimación dinámica satisfactoria; en este estudio se realizaron en promedio 1.8 procedimientos con un fin dinámico ${ }^{4,15,17,19,24-26}$. Todos los pacientes sometidos a procedimientos dinámicos recibieron rehabilitación posquirúrgica con terapia de biofeedback para mejorar la coordinación y los movimientos voluntarios.

El procedimiento dinámico más frecuente en este estudio fue el colgajo libre de gracilis, seguido de los injertos nerviosos cruzados como primer tiempo para el colgajo de gracilis y como procedimiento dinámico aislado para la parálisis facial con una evolución de menos de 6 meses. En la actualidad, los procedimientos dinámicos son el tratamiento de elección para la reconstrucción de la dinámica facial, ya que los resultados son más exitosos y fructíferos que con los procedimientos estáticos, y deberán ser considerados como primera opción a menos que exista alguna contraindicación o comorbilidad que no permita su realización. Como limitaciones de estos procedimientos cuando se realizan transferencias musculares libres están la necesidad de un equipo con experiencia en microcirugía, la mayor curva de aprendizaje y el mayor costo. El procedimiento dinámico se elegirá según la etiología de la parálisis facial, el tiempo de evolución, los nervios donadores viables y la musculatura facial viable.

Los procedimientos estáticos consisten en realizar la suspensión de tejidos blandos con el objetivo de lograr una simetría del rostro en reposo, pero sin permitir movimientos voluntarios. Se realizarán dependiendo de las necesidades de cada paciente y como complemento a los procedimientos dinámicos para obtener una mayor satisfacción funcional y estética. Presentan las ventajas de requerir una curva de aprendizaje baja y su menor costo ${ }^{27,28}$. La principal función de los procedimientos estáticos es proporcionar protección corneal y, en menor medida, mejorar la masticación y el habla ${ }^{29}$. En este estudio se realizaron en promedio dos procedimientos con este fin. Dentro de los procedimientos estáticos, el más realizado fue la colocación de una pesa de oro, seguido de la comisuroplastia y la tira tarsal, similar a lo publicado en otras series, en las cuales la mayoría de los procedimientos tienen como fin restablecer la función y la simetría de la zona frontal, periocular y peribucal16,17,19. El procedimiento estático deberá elegirse de acuerdo con la subunidad estética que se desee reconstruir e individualizando el tratamiento en cada paciente.

\section{Algoritmo de tratamiento dinámico del Hospital General Dr. Manuel Gea González}

El Hospital General Dr. Manuel Gea González cuenta con la primera clínica multidisciplinaria para la atención de parálisis facial en México, integrada por cirujanos plásticos, otorrinolaringólogos, oftalmólogos, psicólogos, genetistas, ortodoncistas y fisioterapeutas, y es centro de referencia para cirugía reconstructiva en el país..$^{13}$ Los objetivos del tratamiento consisten en lograr cuatro aspectos fundamentales:

- Simetría facial en reposo.

- Competencia oral y cierre palpebral.

- Movimientos faciales voluntarios con expresión facial espontánea. 


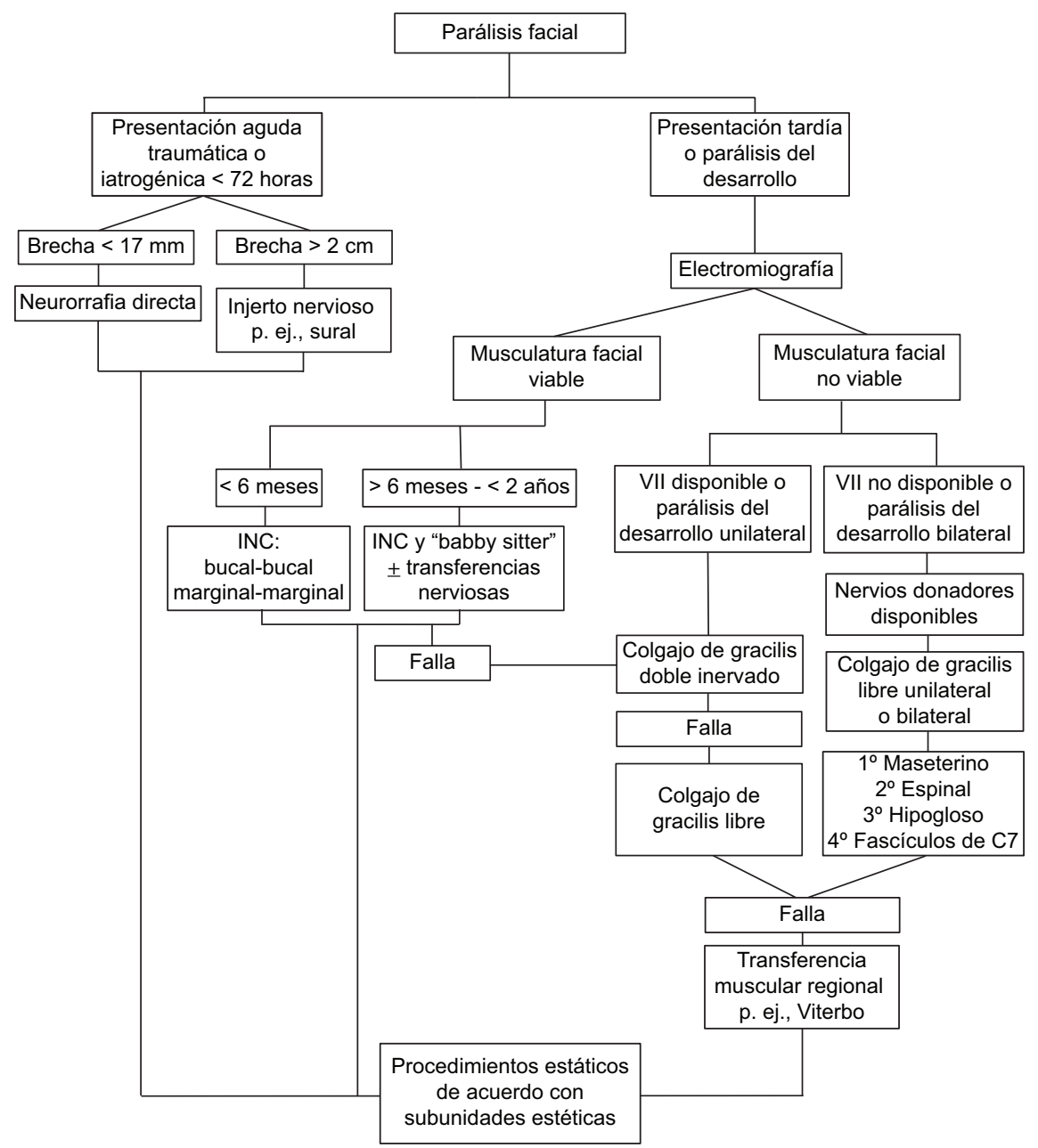

Figura 6. Algoritmo de tratamiento quirúrgico. INC: injerto nervioso cruzado.

- Mínima ausencia de sincinesias y movimientos en masa.

De acuerdo con la experiencia del servicio en el tratamiento de la parálisis facial, proponemos el siguiente algoritmo modificado del propuesto previamente por Terzis y Konofaos ${ }^{17}$ para la reanimación dinámica, dependiendo de la etiología, el tiempo de evolución, los nervios donadores disponibles y el estado de la musculatura facial (Fig. 6).

\section{Presentación aguda $<72$ horas: lesión traumática o iatrogénica (neurorrafia directa)}

Ideal cuando la parálisis se presenta en las primeras 72 horas posterior al trauma o a la lesión iatrogénica. Siempre que sea posible se debe identificar la lesión del nervio facial de ambos cabos e intentar la neurorrafia epineural con dos o tres suturas de nailon 9-0 separadas cuando la brecha sea menor de $17 \mathrm{~mm}$. Si esto no es posible, se deberán usar injertos nerviosos para realizar la neurorrafia libre de tensión. La regla indica que toda brecha mayor de $2 \mathrm{~cm}$ deberá ser reparada con injertos nerviosos. El nervio sural es el principal nervio donador por su fácil disección y baja tasa de complicaciones. Otras opciones son el auricular mayor, el cutáneo antebraquial medial y el cutáneo antebraquial lateral. El tiempo de recuperación promedio es de 12 a 18 meses $^{19,30,31}$.

\section{Presentación tardía: nervio facial homolateral no disponible o parálisis facial del desarrollo}

Previa realización de electromiografía, el tratamiento quirúrgico dependerá del estado de la musculatura facial. 


\section{Musculatura viable para reinervación}

Cuando existe musculatura viable para reinervación, el tratamiento dependerá del tiempo de evolución:

- Lesión de menos de 6 meses: realizar injertos nerviosos-cruzados bucal-bucal o marginal-marginal.

- Lesión de más de 6 meses y menos de 2 años: realizar injertos nerviosos-cruzados y procedimiento baby-sitter (40\% del nervio XII homolateral al VII afectado) con o sin transferencias nerviosas (masetero-bucal e hipogloso-marginal).

\section{Musculatura no viable para reinervación}

En caso de que el estudio neurofisiológico arroje datos de ausencia de actividad muscular, se cuente con una lesión de más de 2 años, parálisis del desarrollo unilateral o bilateral, el tratamiento quirúrgico será una transferencia muscular libre y el nervio donador se elegirá según la disponibilidad de nervios.

\section{Nervio facial contralateral disponible o parálisis del desarrollo unilateral}

En nuestro centro realizamos el colgajo de gracilis doble inervado para la parálisis facial unilateral con nervio facial contralateral disponible en dos tiempos, tal como describieron Cárdenas-Mejía, et al. ${ }^{25}$. El primer tiempo consiste en realizar un injerto nervioso cruzado de bucal sano contralateral hacia el surco nasogeniano de la hemicara afectada, usando el nervio sural. El segundo tiempo se realiza a los 3-4 meses cuando el nervio es viable, confirmado por estudios neurofisiológicos, y consiste en la realización del colgajo muscular libre coaptando el nervio obturador al facial contralateral de manera término-terminal y al nervio maseterino homolateral de manera término-lateral. El tiempo de reinervación promedio es de 8.7 semanas, para los movimientos visibles es de 12.8 semanas y para la recuperación total del edema posquirúrgico es de 7.7 meses. Preferimos esta técnica sobre otras en las que se coapta el nervio maseterino con anastomosis término-terminal al obturador del gracilis y se realiza una anastomosis término-lateral al facial contralateral, porque a pesar de que este nervio provee mayor fuerza y genera una sonrisa más simétrica, raramente logra el objetivo de la espontaneidad $^{32}$. Por otro lado, si solo se realiza el injerto nervioso cruzado del facial contralateral con anastomosis término-terminal al obturador del gracilis se logra mayor espontaneidad, pero con una sonrisa débil y rara vez simétrica $^{33,34}$. La técnica del colgajo de gracilis doble inervado combina estas dos técnicas mediante la estrecha coaptación del nervio maseterino al músculo gracilis, garantizando un tiempo de reinervación más corto, con un mayor número de unidades motoras funcionales, proporcionando más potencia en la activación muscular en menor tiempo, y simultáneamente con una función espontánea e independiente, teniendo mejor resultado sobre otras técnicas descritas. Pese a que en algunas series la edad mayor de 60 años es una contraindicación para realizar este tipo de procedimientos, se han reportado series exitosas de colgajos libres en personas mayores de 65 años $^{35}$.

\section{Nervio facial contralateral no disponible o parálisis del desarrollo bilateral}

En los casos en que el nervio facial contralateral o bilateral no se encuentre disponible, el procedimiento de elección será el colgajo libre de gracilis unilateral o bilateral con microcoaptación al nervio maseterino con anastomosis de la arteria femoral circunfleja a los vasos faciales. Como segunda opción, la coaptación al nervio espinal, y cuando esto no es posible, otras alternativas factibles son el nervio hipogloso o fascículos de $C 7^{17,26}$. Para el síndrome de Moebius entre los 4 y 15 años se iniciaran los procedimientos quirúrgicos con un fin dinámico, previa realización de electromiografía, ya que en un $7.5 \%$ de los casos existen anormalidades ${ }^{26}$. En la mayoría de los casos realizamos el colgajo libre de gracilis bilateral en dos tiempos, con un intervalo de 6 meses $^{24,36}$. Se ha reportado la realización de colgajo libre de gracilis bilateral en un solo tiempo con buenos resultados; en nuestro centro también hemos realizado la transferencia bilateral con esta técnica y representa una opción válida cuando los recursos económicos y el estado de salud del paciente lo permiten ${ }^{37}$.

\section{Falla de los procedimientos libres microquirúrgicos}

Cuando existe fracaso de las transferencias musculares libres, las transferencias regionales son una alternativa factible. El procedimiento más realizado en nuestro centro es la transferencia ortodrómica del músculo temporal descrita por Viterbo y de Paula Faleiros ${ }^{38}$, que se realizó en 11 casos $(9 \%)$ de nuestra serie en los que hubo falla del colgajo de gracilis. 
Aunque se obtienen resultados aceptables en la apariencia general de la cara, particularmente en reposo, la excursión de la sonrisa es inferior en comparación con el resultado obtenido por una transferencia muscular libre. A pesar de esta última limitación, hemos encontrado la transferencia del músculo temporal como una cirugía de rescate confiable ${ }^{38,39}$.

\section{Tercera etapa o cirugías de revisión}

Consiste en realizar cirugías de revisión para mejorar la dinámica y la estática faciales. Estas intervenciones se individualizan dependiendo de la subunidad estética a tratar en cada paciente. Por mencionar algunos de ellos, se pueden realizar colocación de pesa de oro, colgajo minitemporal, neurotización corneal, miomectomía del supresor contralateral, tira tarsal, suspensión de ceja, blefaroplastia asimétrica, ritidectomía asimétrica y lipoinyección facial.

\section{Limitaciones}

Este estudio presenta las limitaciones de ser retrospectivo y no contar con seguimiento a largo plazo de los diferentes procedimientos con escalas validadas de evaluación posoperatorias, pero es el primer estudio de parálisis facial global en México, lo cual abre el camino para próximos estudios.

\section{Conclusiones}

Se requerirán en promedio dos o tres procedimientos quirúrgicos para lograr un resultado estético y funcional aceptable. El procedimiento dinámico se elegirá en función de la etiología, el tiempo de evolución, los nervios donadores disponibles y la musculatura facial viable. El colgajo de gracilis continúa siendo el procedimiento dinámico de elección para la reconstrucción de la parálisis facial; la técnica doble inervado realizada en los casos de parálisis facial unilateral nos ha proporcionado excelentes resultados hasta el momento. Los procedimientos con un fin estático añaden funcionalidad y proporcionan armonía del rostro en reposo. El tratamiento quirúrgico ha logrado mejorar de manera significativa la calidad de vida de los pacientes y deberá ofrecerse en todos los casos.

\section{Agradecimientos}

A todos los autores por el empeño dedicado para la realización de este trabajo, así como al cuerpo del servicio de cirugía plástica y reconstructiva, quienes en su desempeño diario se dedican al manejo óptimo y al bienestar de los pacientes.

\section{Responsabilidades éticas}

Protección de personas y animales. Los autores declaran que para esta investigación no se han realizado experimentos en seres humanos ni en animales.

Confidencialidad de los datos. Los autores declaran que en este artículo no aparecen datos de pacientes.

Derecho a la privacidad y consentimiento informado. Los autores declaran que en este artículo no aparecen datos de pacientes.

\section{Financiamiento}

A cuenta de los mismos autores.

\section{Conflicto de intereses}

Los autores no tienen conflictos de intereses para declarar en relación con el contenido de este artículo.

\section{Bibliografía}

1. Lorch M, Teach SJ. Facial nerve palsy: etiology and approach to diagnosis and treatment. Pediatr Emerg Care. 2010;26:763-73.

2. Guía de práctica clínica, diagnóstico y manejo de la parálisis de Bell (parálisis facial idiopática). México: Secretaría de Salud; 2009.

3. Chuang DC-C, Chang TN-J, Lu JC-Y. Postparalysis facial synkinesis: clinical classification and surgical strategies. Plast Reconstr Surg Glob Open. 2015;3:e320.

4. Terzis JK, Noah ME. Analysis of 100 cases of free-muscle transplantation for facial paralysis. Plast Reconstr Surg. 1997;99:1905-21.

5. Campbell EDR, Hickey RP, Nixon KH, Richardson AT. value of nerve-excitability measurements in prognosis of facial palsy. BMJ. 1962;2:7-10.

6. Telich-Tarriba JE, Cárdenas-Mejía A. Evaluación electromiográfica del nervio facial y de nervios donadores previo a cirugía de reanimación facial en pacientes con síndrome de Moebius. Cir Cir. 2020;88(1).

7. Gantz BJ, Holliday M, Gmuer AA, Fisch U. Electroneurographic evaluation of the facial nerve. Ann Otol Rhinol Laryngol. 1984;93:394-8.

8. Baugh RF, Basura GJ, Ishii LE, Schwartz SR, Drumheller CM, Burkholder R, et al. Clinical practice guideline: Bell's palsy. Otolaryngol Head Neck Surg. 2013;149(3 Suppl):S1-S27.

9. Ishii L, Godoy A, Encarnacion CO, Byrne PJ, Boahene KDO, Ishii M. Not just another face in the crowd: society's perceptions of facial paralysis. Laryngoscope. 2012;122:533-8.

10. Constantinides M, Doud Galli SK, Miller PJ. Complications of static facial suspensions with expanded polytetrafluoroethylene (ePTFE). Laryngoscope. 2001;111:2114-21.

11. Razfar A, Lee MK, Massry GG, Azizzadeh B. Facial paralysis reconstruction. Otolaryngol Clin North Am. 2016;49:459-73.

12. Luijmes RE, Pouwels S, Beurskens CHG, Kleiss IJ, Siemann I, Ingels KJAO Quality of life before and after different treatment modalities in peripheral facial palsy: a systematic review. Laryngoscope. 2017;127:1044-51.

13. Telich-Tarriba JE, Velazquez E, Theurel-Cuevas A, Shinji-Perez K, Anaya-Ayala JE, Jimenez-Murat $Y$, et al. Upper extremity patterns of injury and management at a plastic and reconstructive surgery referral center in Mexico City. Ann Plast Surg. 2018;80:23-6.

14. Arrieta-Joffe $P$, Pérez-Dosal M, Ortiz-De-Zarate G, Cárdenas-Mejía, A Estudio clínico, citogenético, molecular y de imagen de los pacientes con síndrome de Moebius del Hospital General "Dr. Manuel Gea González", Ciudad de México. Cir Plast Iberolatinoam. 2017;43:395-400.

15. Palafox D, Arrieta-Joffe $P$, Cárdenas-Mejía A. Tratamiento quirúrgico reconstructivo actual del síndrome de Moebius. Cirugía Plástica. 2014;24:136-44. 


\section{E. Chávez-Serna, et al.: Parálisis facial, causas y tratamiento quirúrgico en un tercer nivel de atención}

16. Gousheh J, Arasteh E. Treatment of facial paralysis: dynamic reanimation of spontaneous facial expression - apropos of 655 patients. Plast Reconstr Surg. 2011;128:693e-703e.

17. Terzis JK, Konofaos P. Experience with 60 adult patients with facial paralysis secondary to tumor extirpation. Plast Reconstr Surg. 2012:130:51e-66e.

18. Vakharia K, Vakharia K. Bell's palsy. Facial Plast Surg Clin North Am. 2016;24:1-10

19. Hohman $\mathrm{MH}$, Hadlock TA. Etiology, diagnosis, and management of facia palsy: 2000 patients at a facial nerve center. Laryngoscope. 2014;124:E283-93.

20. Gaudin RA, Jowett N, Banks CA, Knox CJ, Hadlock TA. Bilateral facia paralysis. Plast Reconstr Surg. 2016;138:879-87.

21. Cha $\mathrm{Cl}$, Hong CK, Park MS, Yeo SG. Comparison of facial nerve paralysis in adults and children. Yonsei Med J. 2008;49:725

22. Nash JJ, Friedland DR, Boorsma KJ, Rhee JS. Management and outcomes of facial paralysis from intratemporal blunt trauma: a systematic review. Laryngoscope. 2010;120:1397-404.

23. Evans AK, Licameli G, Brietzke S, Whittemore K, Kenna M. Pediatric facial nerve paralysis: patients, management and outcomes. Int J Pediatr Otorhinolaryngol. 2005;69:1521-8.

24. Zuker RM, Goldberg CS, Manktelow RT. Facial animation in children with Möbius syndrome after segmental gracilis muscle transplant. Plast Reconstr Surg. 2000;106:9.

25. Cardenas-Mejia A, Covarrubias-Ramirez JV, Bello-Margolis A, Rozen S. Double innervated free functional muscle transfer for facial reanimation. J Plast Surg Hand Surg. 2015;49:183-8.

26. Cardenas-Mejia A, Palafox D. Facial reanimation surgery in Möbius syndrome: experience from 76 cases from a tertiary referral hospital in Latin America. Ann Chir Plast Esthet. 2018;63:338-42.

27. Hadlock TA, Greenfield LJ, Wernick-Robinson M, Cheney ML. Multimodality approach to management of the paralyzed face. Laryngoscope. 2006;116:1385-9.
28. Hadlock T. Evaluation and management of the patient with postoperative facial paralysis. Arch Otolaryngol Head Neck Surg. 2012;138:505

29. White $H$, Rosenthal E. Static and dynamic repairs of facial nerve injuries. Oral Maxillofac Surg Clin North Am. 2013;25:303-12.

30. Millesi H. Nerve grafting. Clin Plast Surg. 1984;11:115-20.

31. Orgel MG. Epineurial versus perineurial repair of peripheral nerves. Clin Plast Surg. 1984;11:105-13.

32. Biglioli F, Colombo V, Tarabbia F, Pedrazzoli M, Battista V, Giovanditto F, et al. Double innervation in free-flap surgery for long-standing facial paralysis. J Plast Reconstr Aesthet Surg. 2012;65:1343-9.

33. Bae Y-C, Zuker RM, Manktelow RT, Wade S. A comparison of commissure excursion following gracilis muscle transplantation for facial paralysis using a cross-face nerve graft versus the motor nerve to the masseter nerve. Plast Reconstr Surg. 2006;117:2407-13.

34. Manktelow RT, Zuker RM. Cross-facial nerve graft - the long and short graft: the first stage for microneurovascular muscle transfer. Oper Tech Plast Reconstr Surg. 1999;6:174-9.

35. Hembd A, Harrison B, Rocha CSM, Rocha FS, Chamseddin K, Labbé D, et al. Facial reanimation in the seventh and eighth decades of life. Plast Reconstr Surg. 2018;141:1239-51.

36. Klebuc MJA. Facial reanimation using the masseter-to-facial nerve transfer. Plast Reconstr Surg. 2011;127:1909-15.

37. Lu JC-Y, Chuang DC-C. One-stage reconstruction for bilateral Möbius syndrome. Ann Plast Surg. 2013;70:180-6.

38. Viterbo $F$, de Paula Faleiros HR. Orthodromic transposition of the temporal muscle for facial paralysis: made easy and better. J Craniofac Surg. 2005;16:306-9.

39. Garcia RM, Hadlock TA, Klebuc MJ, Simpson RL, Zenn MR, Marcus JR Contemporary solutions for the treatment of facial nerve paralysis. Plast Reconstr Surg. 2015;135:1025e-1046e. 